\title{
A Model for Energy Usage in IEEE 802.11s Based Mesh Networks
}

\author{
VEENU MOR* and HARISH KUMAR \\ University Institute of Engineering and Technology, Panjab University, Chandigarh 160 036, India
}

(Received on 09 January 2018; Revised on 26 June 2018; Accepted on 27 June 2018)

\begin{abstract}
For wireless communications numbers of standards are there in market. Among these standards, IEEE 802.11s is specifically designed for Wireless Mesh Networks (WMN). Mesh nodes in IEEE 802.11s can maintain link specific power mode, independent of power saving mode (PSM) for other peers. In environment having scarce resource of energy, every joule can be precious. This work focuses on analysis of PSM of IEEE 802.11s in view of energy, delay and throughput. Energy consumption due to link-based PSM has been mathematically modelled and compared with existing work. Impact of number of peer links, beacon period, delivery traffic indication map period, bandwidth, traffic, awake window, etc., over energy efficiency have been analysed in light sleep and deep sleep mode. It is observed that, due to more number of peer links in denser network, light sleep mode is less effective in term of energy efficiency. WMN have scalability issue. Further, network nodes near to gateway have lesser lifetime compared to other nodes, but due to PSM significant increase in lifetime has been observed. With increasing traffic, a node devotes more in awake state, so network throughput does not degrade due to light sleep mode. But at lower Beacon Interval slight degradation in throughput has been observed. During low traffic period, ideal listening dominates. Hence, suitable configuration of parameters like awake window, beacon period and delivery traffic indication map period can play vital role in energy saving.
\end{abstract}

Keywords: Energy Conservation; Link Based Power Saving Mode; IEEE 802.11s; Redundantlinks

\section{Introduction}

Institute of Electrical and Electronic Engineers (IEEE) has established various transmission standards like Wireless Personal Area Networks (WPAN) (IEEE 802.15), Wi-Fi (IEEE 802.11), Wireless Metropolitan Area Networks (WMAN) (IEEE 802.16), Cellular Network or Wide Area Network (WAN) (IEEE 802.20, IEEE 802.21), etc. to support mobility in wireless network. Being different in connection speed and radio frequency, each one is more suitable to a specific area and has its own boundaries.

- WPAN is intended for very short range region ( 10 meters). They are generally controlled by a single person for example to transfer files among two handheld devices.

- $\quad \mathrm{Wi}-\mathrm{Fi}$ is known for wireless connectivity over local area, an alternative to wired local area network. Wi-Fi has limited coverage area but it has higher data rate than WPAN.
- Wi-Max is designed for long-range networking (spanning miles or kilometres) as opposed to WLAN and has high data rate but need line of sight communication.

- WAN is suitable for wider range to connect across metropolitan, regional, national or international boundaries using leased telecommunication lines. It has low data speed as compared to Wi-Max and Wi-Fi.

With increasing demand of Internet access service, Wireless mesh networks (WMN) have been emerged to overcome limitation of existing one. WMN can be realized with combinations of various wireless technologies like WPAN, Wi-Fi, WMAN, WAN, etc. and are Interoperable. Wireless connectivity at backhaul part makes them fast to deploy, expandable, cost effective and self configurable.

WMN are becoming favoured network and it is important to analysis energy consumption aspects. At

*Authorfor Correspondence: E-mail:veenu.mor@pu.ac.in, hariskk@pu.ac.in 
access part WMN client nodes have limited storage capacity due to size of battery storage. While at backhaul part, mesh router may have been deployed in difficult areas like battlefield etc., where power cable is not available hence making mesh routers (MR) battery operated. For WMN deployed in rural area, energy can be a limited resource. In other cases, MR may be dependent on unreliable energy resource e.g. renewable energy sources like wind and solar power. So, power management is a major factor for $24 \times 7$ availability of Internet service via wireless mesh network. In such scenario each unit of energy can be proved very valuable. Keeping in view of interest in WMN, IEEE is working on base standards. Traditional IEEE 802.11 MAC layer had been designed for single hop network, its direct application in WMN results in performance degradation. To fill this gap, IEEE 802.11 MAC amendment as per needs of multi hop relaying has been achieved in IEEE 802.11s. Further, IEEE 802.15.5 is an enhancement of IEEE 802.15 to support mesh networking and IEEE 802.16j has been developed for Wi-Max multi hop communication.

This paper focuses on energy saving aspects of IEEE 802.11s (IEEE Standard for Information Technology, 2011). The standard specifies the power management scheme to address the energy efficiency issue in the Medium Access Control (MAC) layer. Unlike previous standards, IEEE 802.11s mesh node can maintain peer specific power mode. IEEE $802.11 \mathrm{~s}$ doesn't explain triggering of power saving mode (PSM). Energy requirement in rural/battlefield environments is dominated by listening mode. Energy consumption due to idle peer listening should be minimized. Until now only few research works are found to focus in the area of IEEE 802.11s. To the best of our knowledge energy performance of peer specific PSM of links due to control packets and data is not fully explored yet.So it is hard to evaluate PSM of IEEE 802.11s. In this research, the focus is to propose a mathematical model for analysis of energy efficiency. It gives accuracy and prepares the way for better control of a system. With thorough understanding of the system modelled, it can be useful for scientist to choose optimal configuration parameters like peer links, beacon interval, Delivery traffic indication map (DTIM) period, queue buffer size, bandwidth, awake window, node density, etc.

The main contributions of the paper are summarized as follows:

- A mathematical model has been proposed to evaluate energy consumption due to beacons and traffic under PSM.

- Energy consumption due to scalability in number of peer links, beacon interval, DTIM period, bandwidth, awake window, node density, network life time, etc. has been analysed.

- Throughput and delay has been evaluated in PSM and results have been validated with test bed observations.

The remaining of text is planned as follows. In section 2, related area of work is covered. Section 3 presents a detailed step by step description of power management in IEEE 802.11s. Mathematical modelling for energy consumptions due to peer links is proposed in section 4. Numerical results and discussion are given in section 5. Comparison with existing work is given in section 6. Finally, concluding remarks and future direction are presented in section 7 .

\section{Related Works}

Extensive works have been made on the energy efficient performance analysis of IEEE 802.11 standard. An analytical model for PSM of IEEE 802.11 has been proposed in (Swain et al., 2015). Under different traffic conditions, network parameters like throughput, estimated delay and expected energy consumption have been analysed. Further, (Aydogdu et al., 2011) have performed analytical study to evaluate energy efficiency of IEEE 802.11 in distributed coordination function with respect to collisions, retransmission, overhearing, etc. Powers saving schemes that have been proposed so far generally took up the following aspects of IEEE 802.11 PSM: setting best possible value for ATIM window size (Jangsoo and Sungchun, 2009), listening (Kindt and Chakraborty, 2018) and beacon interval (Swain et al., 2015) for realistic sleep time; efficient medium-access control for collision avoidance (Miao et al., 2016; Shahin et al., 2018) traffic shaping to extend sleep duration of nodes (Rodenas-Herraiza $e t$ al., 2015); optimizing value of timer to wakeup doze node in delay tolerant traffic and hence can achieve ideal doze duration within acceptable quality of service(Zhu, 2015); dynamic adaptation of link rate 
as per current network condition (Purandare, 2016); scheduling of contention-free time slots for communication among Mobile Station (MS) to reduce competition to access channel and hence minimize collisions. The approach minimize number of MS awake at same time (Rahimi Malekshan, 2016) etc.

Though, IEEE 802.11s is an extension of IEEE 802.11 , but results of IEEE 802.11 cannot be directly applied to IEEE $802.11 \mathrm{~s}$. Keeping this in view researchers have started focusing on analysis of IEEE 802.11s in recent years. In (Lei et al., 2012) authors have detailed performance impact of Request to Send (RTS) frame collisions and data frame collisions. Similarly in other research work (Carrano et al., 2013) a thorough analysis of advantages and disadvantages of IEEE 802.11s and focus to the path discovery mechanism is considered. Both studies (Carrano et $a l ., 2013$ ) and (Lei et al., 2012) have completely ignored energy consumption analysis. Challenges of IEEE $802.11 \mathrm{~s}$ draft 2.02 versions at MAC layer have been presented in (Sgora et al., 2009). A broad overview of aspects like beaconing, congestion control, power management etc., is given. But PSM is discussed only at elementary level. In another work (Bauschert and Porsch, 2012) authors have analysed the performance of PSM of IEEE 802.11sstandard (IEEE Standard for Information Technology, 2011). Test bed implementation acknowledges that roundtrip time (RTT) and throughput degrades in PSM. RTT linearly increases with the beacon interval of the nodes in PSM. Another research (Bauschert and Porsch, 2013) have evaluated that scalability of IEEE 802.11 s degrades under power saving schemes. Further, subsequent work by (Bauschert and Porsch, 2014) have overcome scalability issues of IEEE 802.11 s in light sleep mode by proposing a harmonizing scheme. Antonio et al., (2012) have considered energy saving in WMN with devices equipped with multiple network interfaces. Traffic of nearby links doesn't interfere with nearby. Links in backhaul have fixed bidirectional capacity. While (Ma et al., 2014) have reduced energy consumption by link scheduling based on concept of allocating slots in continuation for incident links. But in proposed scheduling a few of the slots may not be utilized properly. It is assumed that each incident link needs one slot at a given time and lacks consideration of varying traffic demands on links. Until now only few works are found to focus in the area of IEEE 802.11s. Additionally, link specific PSM of IEEE 802.11s has not been addressed adequately.

\section{Power Management in IEEE 802.11s}

IEEE 802.11s mesh node can maintain link specific power mode, independently of PSM for others. A mesh node can operate in three power states- active, light sleep and deep sleep mode towards its peers as summarized in Table 1. Peer node is a node to which a mesh peers i.e., direct communication can be established. Beacons are kind of packets sent by mesh nodes for synchronization. Unlike earlier versions of IEEE 802.11, each mesh node in IEEE 802.11s has their own Target Beacon Transmission Times (TBTT). If a node is in light sleep mode towards a peer node, it enters in awake state prior to every TBTT of the corresponding peer node to receive its beacon frame. Whereas a node in deep sleep mode towards peer doesn't need to wake up to listen all peer beacons. But whether a mesh node is in light or deep sleep mode, it wakes up at every TBTT of its own and stay in awake state for duration of mesh awake window. While a node in active mode towards a peer stay awake all the time and listens to all peer beacons and hence withdraws maximum energy.

Table 1. Power Saving Modes in IEEE 802.11s

\begin{tabular}{|c|c|}
\hline Power Saving Mode & Peer Station \\
\hline Active Mode & $\begin{array}{l}\text { - Awake all time } \\
\text { - Power consumption is highest }\end{array}$ \\
\hline \multirow[t]{3}{*}{ Light Sleep Mode } & $\begin{array}{l}\text { - Attentive to all beacons from peer } \\
\text { mesh STA }\end{array}$ \\
\hline & $\begin{array}{l}\text { - Alternates between active and } \\
\text { sleep mode }\end{array}$ \\
\hline & $\begin{array}{l}\text { - Power consumption is lesser than } \\
\text { active mode }\end{array}$ \\
\hline \multirow[t]{3}{*}{ Deep Sleep Mode } & $\begin{array}{l}\text { May not be attentive to all the } \\
\text { beacons from the corresponding } \\
\text { peer mesh STA }\end{array}$ \\
\hline & $\begin{array}{l}\text { - Alternates between active and } \\
\text { sleep mode }\end{array}$ \\
\hline & $\begin{array}{l}\text { - Power consumption is least among } \\
\text { all three modes }\end{array}$ \\
\hline
\end{tabular}

Various PSM for peer nodes are illustrated in Fig. 1. For three states, ${ }^{3} \mathrm{P}_{2}$ possible combinations exist. Six nodes are considered, each node is having 2 peer nodes for this scenario. 


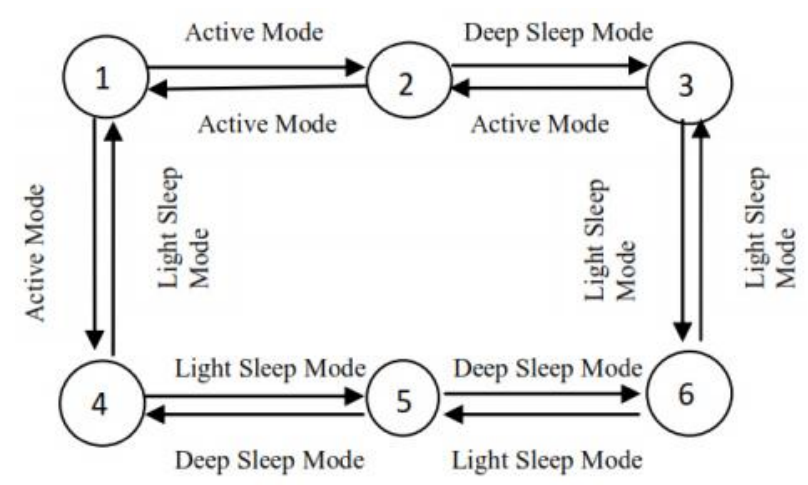

Fig. 1: Various power saving combinations for IEEE 802.11s

- $\quad$ Node (1) is in active mode for peer $(2,4)$.

- $\quad$ Node (2) is in active mode towards peer (1) and deep sleep mode towards peer (3).

- $\quad$ Node (3) is in light sleep mode towards peer (6) and active mode towards peer (2).

- $\quad$ Node (4) is in light sleep mode towards peer (1, $5)$.

- Node (5) is in deep sleep mode towards peer $(4,6)$.

- Node (6) is in light sleep mode towards node (3, $5)$.

Among all nodes (5) is working in most power conservative mode. Referring IEEE 802.11s standard draft (IEEE Standard for Information Technology, 2011), nodes $(4,5,6)$ are not in active mode for peer nodes hence may toggle to doze state i.e., can switch off their transmitters. Similarly, nodes $(1,2,3)$ acts in active mode for at least one of their peer nodes and doesn't satisfy criteria for entering doze state, hence remains in awake state. For maximum power saving, power change mode criteria should switch a node in light or deep sleep mode for all its peer nodes. In parallel careless handling of PSM may also deteriorate quality of service. Working of various PSM for peer nodes can be understood from Fig. 2 to Fig. 5. Fig. 2, illustrates working of two nodes acting in active mode towards each other. Active mode nodes can initiate data transfer without any wait. In Fig. 3, node (4) is in light sleepmode towards node (1). So node (1) can't initiate data transmission at link $(1,4)$ arbitrary and has to wait till node (4) wakes up to listen beacon of node (1). Fig. 4 represents a node in deep sleep mode doesn't need to wake up to listen to beacon of peer

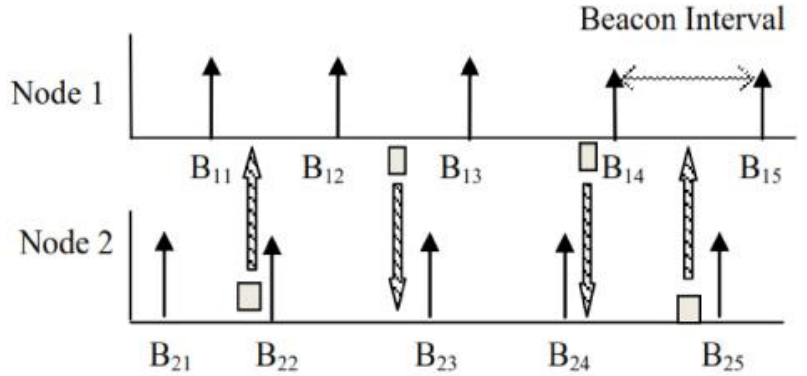

Fig. 2. Nodes (1) and (2) are in active mode to each other

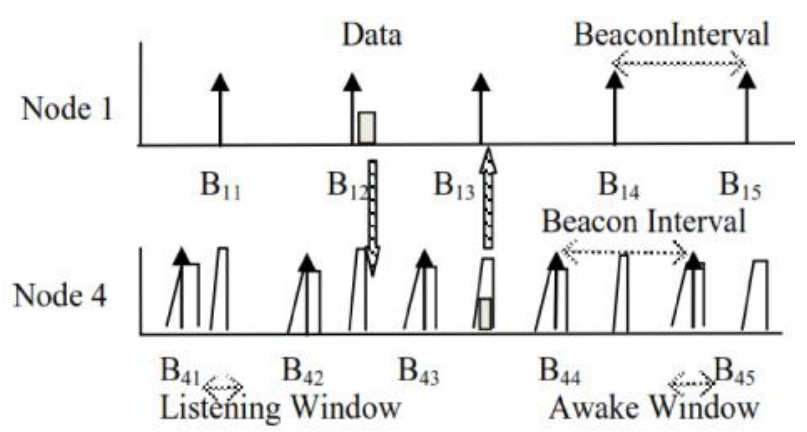

Fig. 3: Node (1) is in active mode towards node (4) and in light sleep mode vice-versa

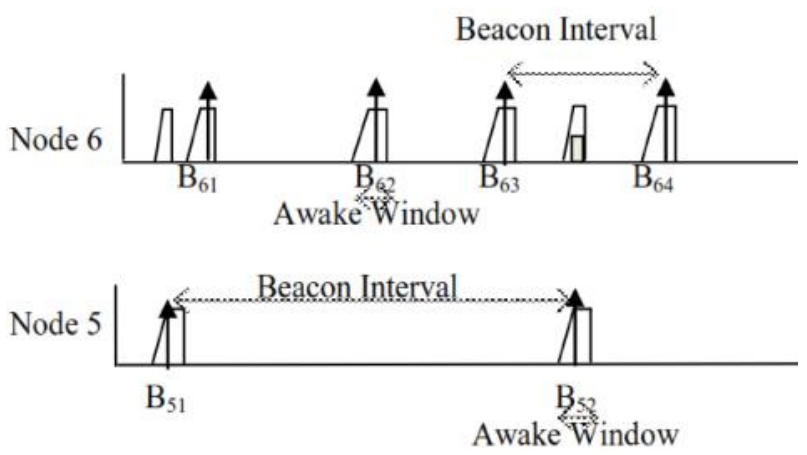

Fig. 4: Node (6) is in light sleep mode towards node (5) and in deep sleep mode vice-versa

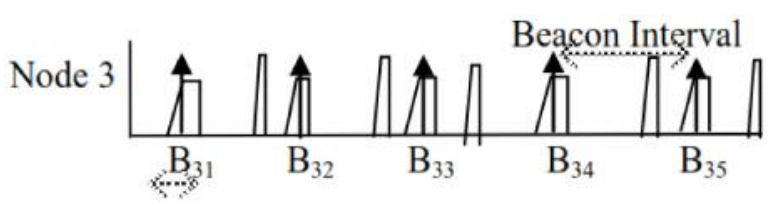

Awake Window

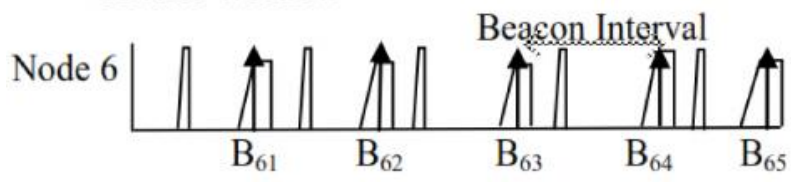

Fig. 5: Nodes $(3,6)$ are in light sleep mode towards each other 
nodes. While, Fig. 5 shows a node in light sleep mode has to wake up every time at TBTT of corresponding peer nodes.

\section{Proposed Model for Measuring Energy Consumption}

Power consumption of a node in PSM can be determined by many factors. IEEE $802.11 \mathrm{~s}$ draft doesn't specify PSM alter criteria for switching a node in light or deep sleep mode with respect to peer links. To help network nodes to work for longer duration, these may choose to switch to light sleep mode when renewable power supply is not available or can change to deep sleep mode when power level of battery drops below a threshold value.

According to $802.11 \mathrm{~s}$ specification if a node has its entire peer in light/deep sleep mode only then it can be switched to doze state. Nodes in PSM wakes up to listens beacon of peer and to transmit that of their own. As focus of this work is to analyse energy consumption due to beaconing, in this section the energy consumption due to beacons and traffic have been modelled. Impact of number of peer links and traffic load on network life time have been revealed. Section (4.1) and (4.2) models energy consumption in receiving and transmitting beacons under no traffic respectively. Section 4.3 gives total energy consumption in light and deep sleep mode in void traffic. Fig. 6 summarizes energy consumption in power save mode. The model is further extended to give energy consumption under traffic in section (4.4). Section (4.5) concludes total save in energy under light sleep mode relative to active mode. Section (4.6) models the uniform traffic distribution, to enhance lifetime of whole network. It is observed that with increase in number of peer links net save in energy decreases. The numerical analysis for various parameters has been given in Section 5 .

\section{Energy Consumption in Receiving Beacons in Power Save Mode}

In deep sleep mode a node doesn't need to receive all beacons of its peer links. But in light sleep mode a node wakes up to receive all beacons of peers. For a given node (i), energy is drawn for listening beacon of peer nodes. This can be collectively determined by energy consumed in receiving a beacon from a peer node $\left(E_{b}^{R}\right)$, switching energy from sleep mode to active mode $\left(\mathrm{E}_{\mathrm{LA}}\right)$ and idle energy while waiting for peer beacon $\left(\mathrm{R}_{\mathrm{awk}}\right)$. Energy consumption in receiving beacons for each beacon of peer link is given in (1). With average number of ' $k$ ' peer links, (1) can be rewritten as in (2).

$$
\begin{aligned}
& \mathrm{E}_{\mathrm{iR}}^{\prime \prime}=\left(\mathrm{E}_{\mathrm{b}}^{\mathrm{R}}+\mathrm{E}_{\mathrm{LA}}+\mathrm{R}_{\mathrm{awk}}\right) \\
& \mathrm{E}_{\mathrm{iR}}=\mathrm{k}\left(\mathrm{E}_{\mathrm{b}}^{\mathrm{R}}+\mathrm{E}_{\mathrm{LA}}+\mathrm{R}_{\mathrm{awk}}\right)
\end{aligned}
$$

$\mathrm{E}_{\mathrm{b}}^{\mathrm{R}}$ linearly depends on power used in receiving a beacon and receiving time as in (3) and (4).

$$
\begin{aligned}
& \mathrm{E}_{\mathrm{b}}^{\mathrm{R}}=\mathrm{P}_{\mathrm{r}} \mathrm{T} \text { or } \\
& \mathrm{E}_{\mathrm{b}}^{\mathrm{R}}=\mathrm{P}_{\mathrm{r}} \frac{\mathrm{S}}{\mathrm{B}}
\end{aligned}
$$

where,

$$
\begin{aligned}
& \mathrm{P}_{\mathrm{r}}=\text { Power used in receiving a beacon } \\
& \mathrm{T}=\text { Transmission time for beacon } \\
& \mathrm{S}=\text { Beacon size } \\
& \mathrm{B}=\text { Bandwidth }
\end{aligned}
$$

Power received at receiver decays with distance between transmitter and receiver. It depends upon path loss exponent $\alpha$. Transmission power should be chosen such that received power is above threshold value for successful decoding.

IEEE $802.11 \mathrm{~s}$ implementation inherently depends on IEEE $802.11 \mathrm{a} / \mathrm{b} / \mathrm{g}$ etc. The setup time for other circuit blocks is insignificant compared to that of the frequency synthesizers. Thus, $\mathrm{E}_{\mathrm{LA}}$ is dominated by that of frequency synthesizers. Energy consumption due to switching from sleep mode to active mode can be calculated by (5). Factor $\left(\mathrm{I}_{\text {active }}-\mathrm{I}_{\text {sleep }}\right)$ considers the fact of current leakage in sleep mode, $\mathrm{I}_{\text {sleep }}$ may not be zero and $\left(\frac{\mathrm{V}}{2}\right)$ is average of voltage while switching from sleep to active mode state. 


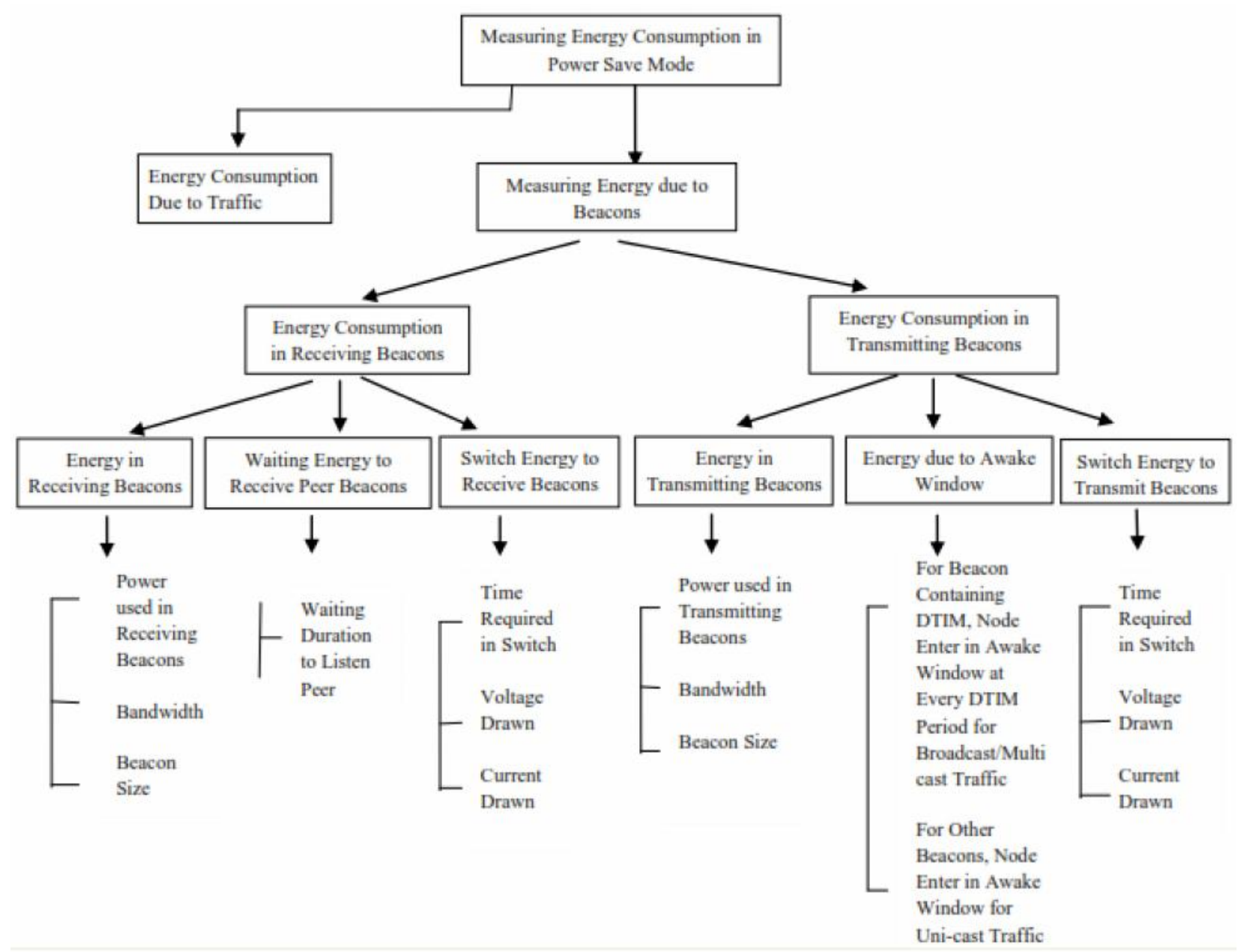

Fig. 6: Measuring energy consumption in power save mode

$$
\mathrm{E}_{\mathrm{LA}}=\left(\mathrm{I}_{\text {active }}-\mathrm{I}_{\text {sleep }}\right) \mathrm{F} \frac{\mathrm{V}}{2}
$$

where,

$I_{\text {active }}=$ Current drawn in active mode

$I_{\text {sleep }}=$ Current draw in sleep mode, this may be negligible in comparison to $I_{\text {active }}$

$\mathrm{F}=$ Time required for the radio in switching from sleep to active mode

$\mathrm{V}=$ Voltage drawn

$\mathrm{R}_{\text {awk }}$ signifies energy consumed while in listening mode for peer beacons. A node in PSM wakes up before TBTT by $\mathrm{R}_{\text {margin }}$ time and wait for beacon latest by $\mathrm{R}_{\text {expiry }}$ time. $\mathrm{R}_{\mathrm{awk}}$ is proportional to wake duration ' $L$ '. After receiving peer beacon, node may switch back to light sleep mode. Peer nodes adjust their TBTT uniformly to avoid beacon collision via Mesh Beacon Collision Avoidance algorithm (IEEE Standard for Information Technology, 2011).

\section{Energy Consumption in Transmitting Beacons in Power Save Mode}

Energy consumption due to transmission of beacons by node (i) is contributed by energy due to transmitting its beacon $\mathrm{E}_{\mathrm{b}}^{\mathrm{T}}$, switching energy from sleep mode to active mode $\mathrm{E}_{\mathrm{LA}}$ and idle energy in awake window. DTIM is a kind of traffic indication map which indicates about the presence of buffered multicast/ broadcast traffic for peer stations. DTIM period determines how regularly a beacon contains DTIM. For beacons other than containing DTIM, a node in light/deep sleep mode enters in awake window only if it has buffered traffic for at least one peer mesh node. For such beacons average energy consumption 
per beacon interval can be calculated by $\mathrm{vD}_{\mathrm{awk}}\left(1-\frac{1}{\text { DTIM Period }}\right)$. Where, $\mathrm{v} \in 0,1$ is a

Boolean variable to represent buffer data for peer nodes and $\mathrm{D}_{\text {awk }}$ is energy consumed in awake window (W). While for every beacons containing DTIM as determined by DTIM period, a node always enters in mesh awake window. Average energy consumption per beacon interval due to awake window of DTIM beacons is given by $\left\{\frac{D_{\text {awk }}}{\text { DTIM Period }}\right\}$. Beacons are transmitted without acknowledgement frame. When DTIM period is 1 , then for every TBTT of its own, node enters in awake window after transmission of its own beacon. Optimizations of DTIM and beacon period can conserve energy in PSM without degrading quality of service. Average energy consumed for transmitting a beacon at a node is given in (6).

$$
\begin{aligned}
\mathrm{E}_{\mathrm{i}} \mathrm{T}= & \left(\frac{\mathrm{D}_{\mathrm{awk}}}{\text { DTIM Period }}+\mathrm{vD}_{\mathrm{awk}}\left(1-\frac{1}{\text { DTIM Period }}\right)\right) \\
& +\left(\mathrm{E}_{\mathrm{b}}^{\mathrm{T}}+\mathrm{E}_{\mathrm{LA}}\right)
\end{aligned}
$$

$\mathrm{E}_{\mathrm{b}}^{\mathrm{T}}$ linearly depends on power used in transmitting a beacon and transmission time as in (7) and (8).

$$
\begin{aligned}
& \mathrm{E}_{\mathrm{b}}^{\mathrm{T}}=\mathrm{P}_{\mathrm{t}} \mathrm{T} \text { or } \\
& \mathrm{E}_{\mathrm{b}}^{\mathrm{T}}=\mathrm{P}_{\mathrm{t}} \frac{\mathrm{S}}{\mathrm{B}}
\end{aligned}
$$

where,

$$
\begin{aligned}
& \mathrm{P}_{\mathrm{t}}=\text { Power used in transmitting a beacon } \\
& \mathrm{T}=\text { Transmission time for beacon } \\
& \mathrm{S}=\text { Beacon size } \\
& \mathrm{B}=\text { Bandwidth }
\end{aligned}
$$

\section{Total Energy Consumption without Traffic}

Using equation (2) and (6), total energy consumption of network due to light sleep mode in void traffic is given by (9) and hence (10).

$$
\begin{aligned}
& \sum_{\mathrm{i}=1}^{\mathrm{N}}\left\{\left(\mathrm{E}_{\mathrm{iR}}+\mathrm{E}_{\mathrm{iT}}\right)\left(\frac{\mathrm{O}}{\mathrm{A}}\right)\right\} \\
& \sum_{\mathrm{i}=1}^{\mathrm{N}}\left\{\left(\mathrm{k}\left(\mathrm{E}_{\mathrm{b}}^{\mathrm{R}}+\mathrm{E}_{\mathrm{LA}}+\mathrm{R}_{\mathrm{awk}}\right)\right\}+\right. \\
& \left(\left(\frac{\mathrm{D}_{\mathrm{awk}}}{\mathrm{DTIM} \text { Period }}\right)+\left(\mathrm{E}_{\mathrm{b}}^{\mathrm{T}}+\mathrm{E}_{\mathrm{LA}}\right)\right)\left(\frac{\mathrm{O}}{\mathrm{A}}\right)
\end{aligned}
$$

In deep sleep mode, node doesn't wake to receive peer beacon, so, total energy consumption of network due to deep sleep mode in void traffic is given by (11) and hence (12):

$$
\begin{aligned}
& \sum_{\mathrm{i}=1}^{\mathrm{N}}\left\{\left(\mathrm{E}_{\mathrm{iT}}\right)\left(\frac{\mathrm{O}}{\mathrm{A}}\right)\right\} \\
& \left\{\left(\frac{\mathrm{D}_{\text {awk }}}{\text { DTIM Period }}\right)+\left(\mathrm{E}_{\mathrm{b}}^{\mathrm{T}}+\mathrm{E}_{\mathrm{LA}}\right)\left(\frac{\mathrm{O}}{\mathrm{A}}\right)\right\}
\end{aligned}
$$

where,

$$
\begin{aligned}
& \mathrm{O}=\text { Duration of observation } \\
& \mathrm{A}=\text { Beacon Interval }
\end{aligned}
$$

\section{Energy Consumption in Traffic}

In this section energy consumption under traffic load has been modelled. Consider a node (i) having k peer nodes with $\mu_{\mathrm{i}}$ Poisson arrival rate per beacon. In 802.11s TBTT of mesh station are evenly distributed to avoid collisions and batch of traffic is scheduled at start of a TBTT in light sleep mode node. Link access can be contention based or TDMA. Contention based access involves two time components one is static due to size of packet another is dynamic for channel acquisition; whereas transmission of packets in TDMA access ensures deterministic access.

Contention Based Access: Total time taken in channel acquisition and transmitting a packet (Fig. 7) in contention based is given by $\mathrm{Pk}^{\mathrm{t}}(13)$ and hence for batch of size $\mu_{\mathrm{i}}$ packets is given by $P k_{\mu_{i}}^{t}$ (14). 


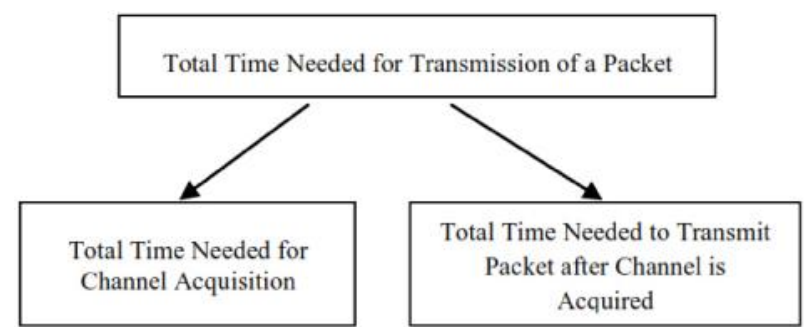

Fig. 7: Total time needed for transmission of a packet

$$
\begin{aligned}
& \mathrm{Pk}^{\mathrm{t}}=\mathrm{T}(\mathrm{S})+\mathrm{U}\left(0, \mathrm{C}_{\text {min }}\right)+\text { DIFS } \\
& \mathrm{Pk}_{\mu_{\mathrm{i}}}^{\mathrm{t}}=\mu_{\mathrm{i}}\left(\mathrm{T}(\mathrm{S})+\mathrm{U}\left(0, \mathrm{C}_{\text {min }}\right)+\text { DIFS }\right)
\end{aligned}
$$

where, $\mathrm{T}(\mathrm{S})=\frac{\operatorname{Packet} \operatorname{Size}\left(\mathrm{P}_{\mathrm{S}}\right)}{\text { Bandwidth }(\mathrm{B})}$ and $\mathrm{U}\left(0, \mathrm{C}_{\text {min }}\right)$ is a uniform random variable depends on contention window size $\mathrm{C}_{\text {min }}$ and contention slot size $\mathrm{C}_{\text {slot }}$. In contention less and noise less network contention window size can be assumed fixed as $\mathrm{C}_{\min }$. For a random variable expected value can be defined as the Lebesgue integral, hence expected value of $U$ is given by (15). DCF Inter-frame Space (DIFS) is time period after which stations can have access to the medium instantly if it is free for the time period longer than the value defined as DIFS.

$$
E(U)=\int_{0}^{X} X P(X) d x=\frac{C_{\text {slot }} C_{\text {min }}}{2}
$$

Average time duration needed for transmission of a packet and hence for batch of size at a node in contention based network is given by (16).

$$
\mathrm{T}_{\mu_{\mathrm{i}}}^{\mathrm{t}}=\mu_{\mathrm{i}}(\mathrm{T}(\mathrm{S})+\mathrm{E}(\mathrm{U})+\mathrm{DIFS})
$$

Deterministic Access: In TDMA, time taken in channel acquisition and transmitting a packet depends on link scheduling algorithm. In contiguous link scheduling (Ma, 2014), traffic for one peer node can be transmitted in continuous slots. Number of required slots for transmission of $\mu_{i}$ packet can be determined from (17). Numerator tells the number of bits to be transmitted over a link in a beacon and denominator gives number of bits that can be transmitted in every slot.

$$
\mathrm{N}_{\mathrm{S}}^{\mathrm{T}}=\frac{\mu_{\mathrm{i}} \mathrm{P}_{\mathrm{S}}}{\mathrm{BD}_{\mathrm{s}}}+\mathrm{H}
$$

where,

$$
\begin{aligned}
& \mathrm{B}=\text { Bandwidth } \\
& \mathrm{P}_{\mathrm{s}}=\text { Packet Size } \\
& \mathrm{D}_{\mathrm{s}}=\text { Duration of slot } \\
& \mathrm{H}=\text { Spacing in slots between transmissions of } \\
& \mu_{1}=\text { Packets to be transmitted }
\end{aligned}
$$
different links

From (17), total time needed for transmission for batch of size $\mu_{\mathrm{i}}$ for TDMA based network is given by (18).

$$
\mathrm{T}_{\mu_{\mathrm{i}}}^{\mathrm{t}}=\mathrm{N}_{\mathrm{S}}^{\mathrm{T}} \mathrm{D}_{\mathrm{s}}+\mathrm{W}^{\mathrm{t}}
$$

Where, $\mathrm{W}^{\mathrm{t}}$ is waiting time for $\mathrm{i}^{\mathrm{th}}$ node to start transmission of $\mu_{\mathrm{i}}$ is given by link scheduling algorithm.

After computing time needed to transmit $\mu_{\mathrm{i}}$ packet in contention based access (16) and contention less access (18), let us compute energy consumption for transmitting and reception for batch of packets as illustrated in Fig. 8 (Cui et al., 2005).

Energy consumption for transmitting a batch of $\mu_{\mathrm{i}}$ packets is given by $\mathrm{EW}_{\mu_{\mathrm{i}}}^{\mathrm{t}}$

$$
\begin{aligned}
& \mathrm{EW}_{\mu_{\mathrm{i}}}^{\mathrm{t}}=\mathrm{E}_{\mathrm{D}} \mathrm{T}_{\mu_{\mathrm{i}}}^{\mathrm{t}}+\mathrm{P}_{\mathrm{t}} \mathrm{T}_{\mathrm{t}} \\
& \mathrm{T}_{\mathrm{t}}=\frac{\mathrm{P}_{\mathrm{S}}}{\mathrm{B}} \mu_{\mathrm{i}}
\end{aligned}
$$

Where, $E_{D}$ is circuit energy consumption per second of a node. $\mathrm{P}_{t}$ is power used in transmitting a packet, $T_{t}(20)$ is time taken in transmitting $\mu_{i}$ packets under bandwidth B. Similarly, energy consumption for receiving a batch of $T_{j, i}$ packets $E_{j, i}^{r}$ is given by (21).

$$
\mathrm{EW}_{\mathrm{j}, \mathrm{i}}^{\mathrm{r}}=\mathrm{P}_{\mathrm{r}} \mathrm{T}_{\mathrm{r}}
$$




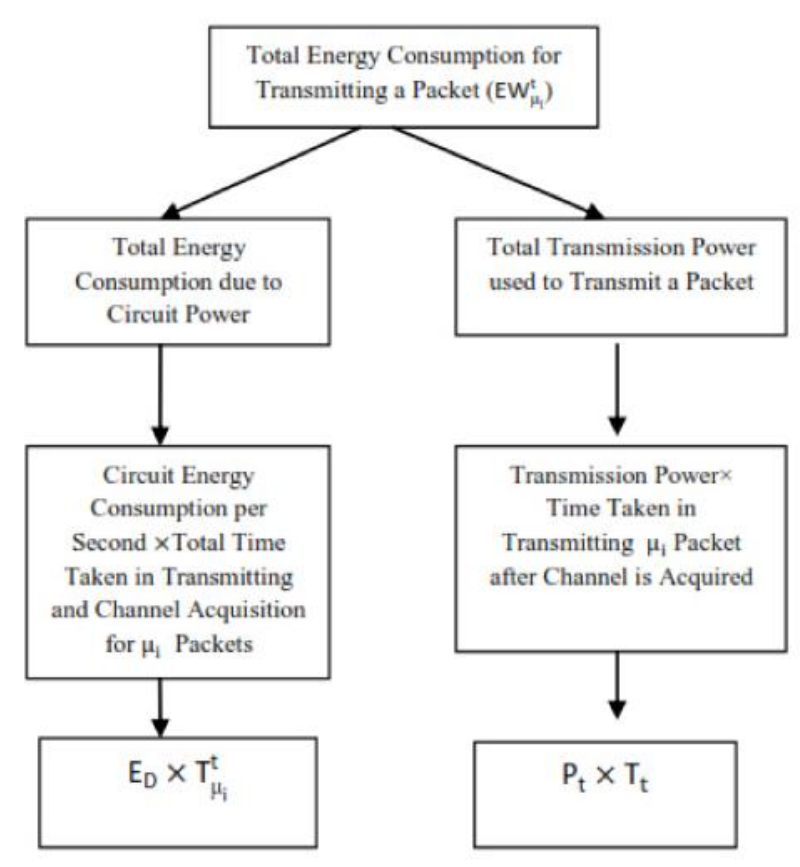

Fig. 8: Total energy needed for transmission of a packet

where, by (22)

$\mathrm{T}_{\mathrm{r}}=$ Time taken in receiving $\mathrm{t}_{\mathrm{j}, \mathrm{i}}$ packets is given

$\mathrm{P}_{\mathrm{r}}=$ Power used by receiving circuit per unit time to receive a packet

$$
T_{r}=\frac{P_{S}}{B} t_{j, i}
$$

Total energy consumed in transmitting $\left(\mathrm{N}_{\mathrm{iT}}\right)$ and receiving $\left(\mathrm{N}_{\mathrm{iR}}\right)$ traffic can be computed using algorithm described by Flowcharts 1 and 2 .

\section{Total Energy Consumption}

Total energy consumption $\mathrm{E}_{\mathrm{iS}}$ per node (i) in light sleep mode is given by (23).

$$
\mathrm{E}_{\mathrm{iS}}=\left(\mathrm{N}_{\mathrm{iR}}+\mathrm{N}_{\mathrm{iT}}\right)\left(\frac{\mathrm{O}}{\mathrm{A}}\right)
$$

where,

$\mathrm{O}=$ Duration of observation

$\mathrm{A}=$ Beacon Interval

For network having $\mathrm{N}$ nodes, total energy

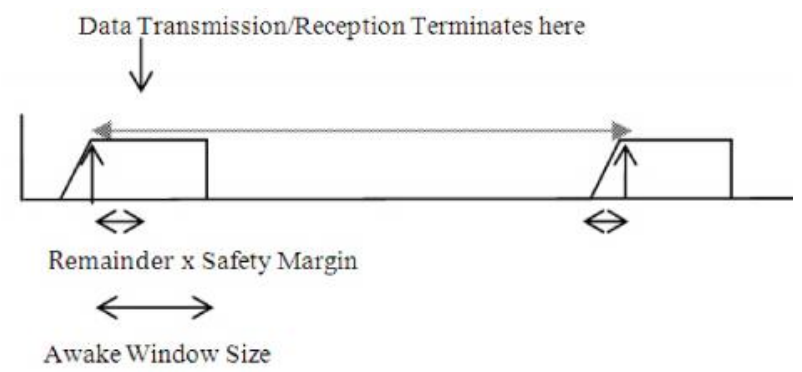

Fig. 9: Data transmission/reception terminates in awake window region

Data Transmission Terminates here

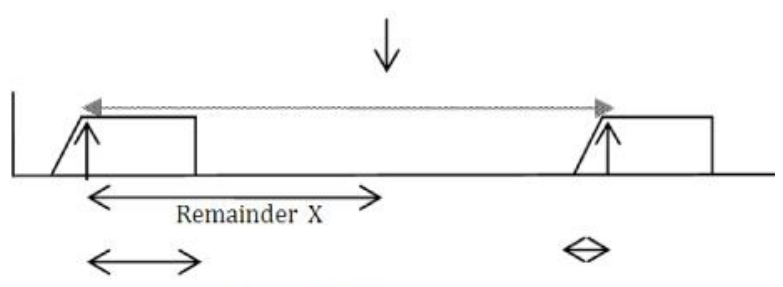

Awake Window Size Safety Margin

Fig. 10: Data transmission/reception terminates in between awake and safety window region

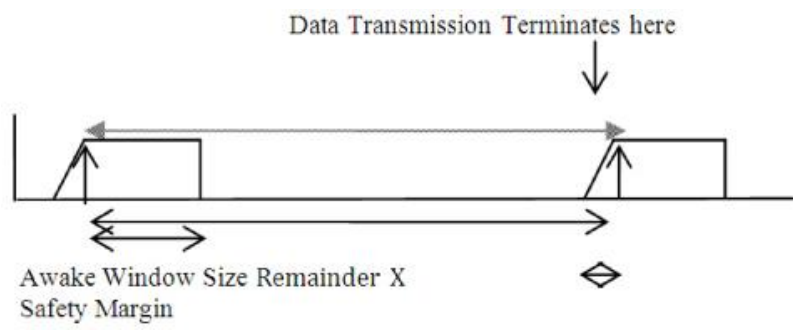

Fig. 11: Ongoing transmission/reception terminates in safety window region of next awake window, and zero remaining time for sleep

consumption of network due to light sleep mode is given by (24).

$$
\mathrm{E}_{\mathrm{S}}=\sum_{\mathrm{i}-1}^{\mathrm{N}} \mathrm{E}_{\mathrm{iS}}
$$

\section{Transmitting and Receiving in Uniform Traffic Distribution}

For sake of clarity, network lifetime analysis has been demonstrated for circular network area, but can be easily extended for other shapes as well. Considering a circular network with radius $R$ and gateway located at centre (Fig. 12). Traffic from mesh nodes are directed towards gateway. In a multi-hop network, 


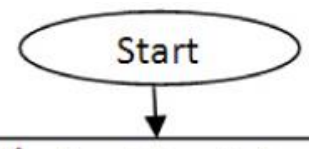

Compute $\mathrm{T}_{\mu_{\mathrm{i}}}^{\mathrm{t}}$, time taken in transmitting $\mu_{i}$ packets using equation (16) or (18)

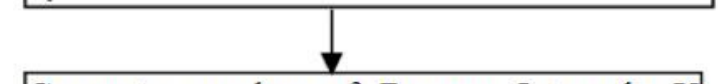

Compute number of Beacon Intervals $\mathrm{X}$ required to transmit $\mu_{\mathrm{i}}$ packets

$\mathrm{X}=\left\lceil\frac{\mathrm{T}_{\mu_{\mathrm{i}}}^{\mathrm{t}}}{\text { Beacon Interval }}\right.$

Compute time required for transmission in last i.e. $\mathrm{N}^{\text {th }}$ Beacon Interval

Remainder $\mathrm{X}=\mathrm{T}_{\mathrm{t}}$ (modulo) Beacon Interval
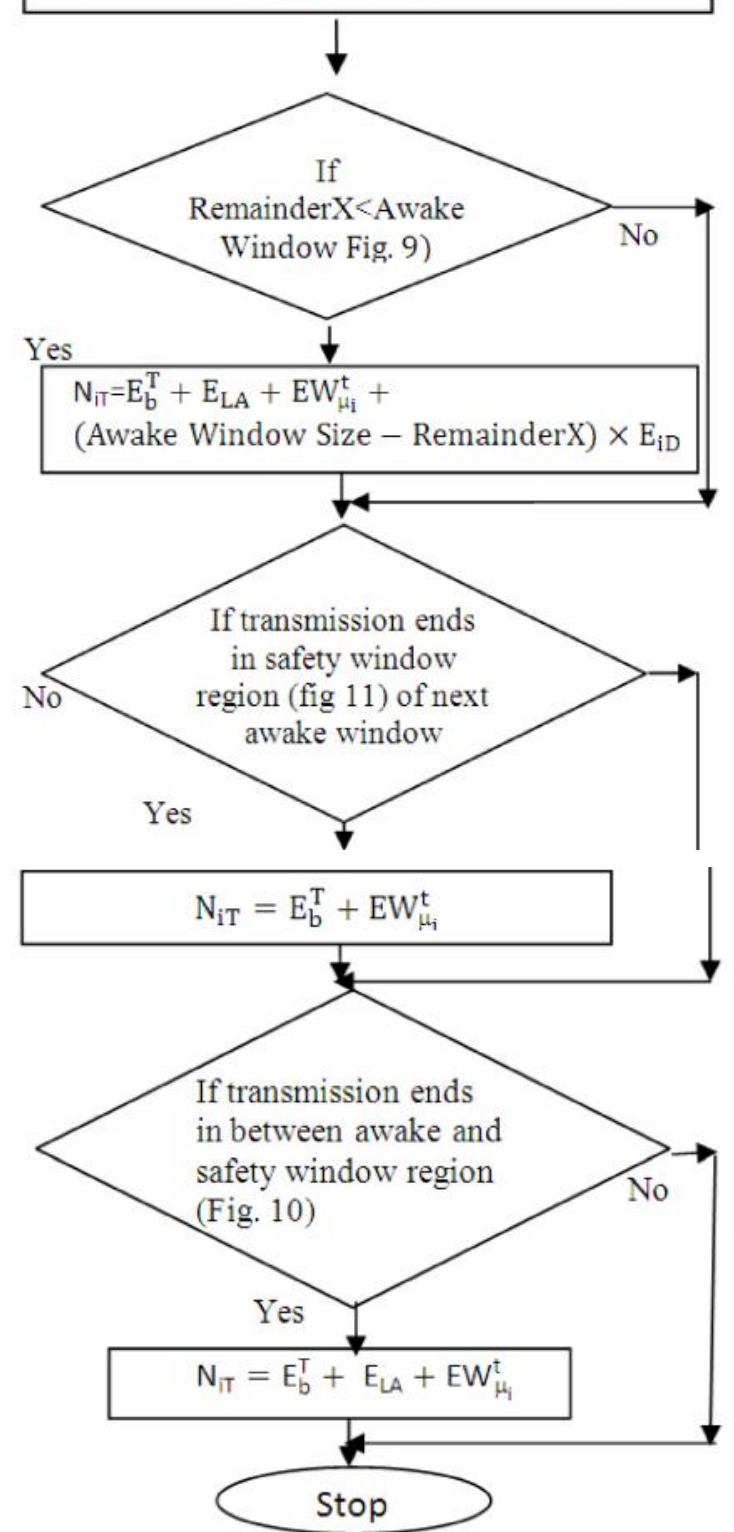

Flowchart 1: Computing energy in transmission data

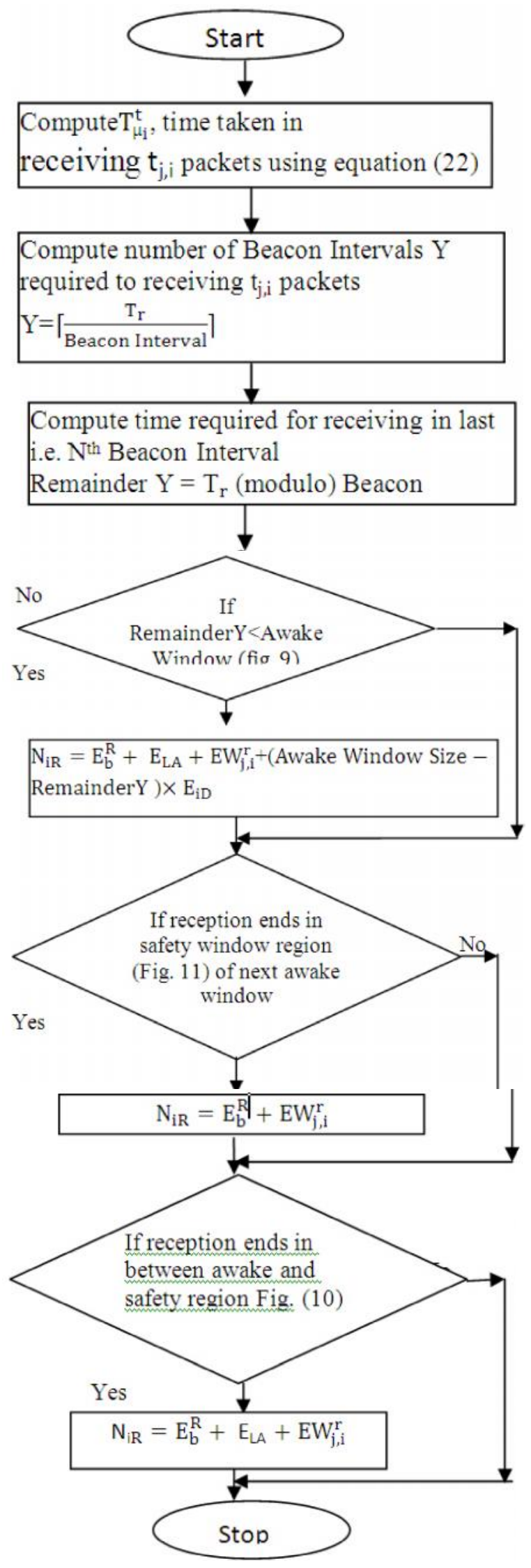

Flowchart 2: Computing energy in receiving data 
routing algorithm determines path trajectory. This in turn decides the life time of a node. More frequently a node used in routing path, sooner it will deplete the energy. So, a node closer to gateway carries more traffic than outer one, and has more energy consumption. So their life time decides of whole network. Keeping this in view lets divide circle into $\mathrm{n}=\left[\frac{\mathrm{R}}{\mathrm{r}}\right]$ rings of width. Mesh nodes are uniformly distributed throughout the area. Among total mesh nodes, density of MAP is â. Rate of traffic generation at MAP in $\mathrm{i}^{\text {th }}$ ring is $\mu_{\mathrm{i}}$ packets per beacon. Area of $\mathrm{i}^{\text {th }}$ ring can be given by $\left(\Pi(\mathrm{ir})^{2}-\Pi((\mathrm{i}-1) \mathrm{r})^{2}\right.$, and area of whole circular network is $\Pi \mathrm{R}^{2}$. So, by definition, probability of a node to be placed in ring is given by (25) and out of $\mathrm{N}$ nodes, number of nodes in ring is.

$$
\mathrm{P}_{\mathrm{i}}=\frac{\Pi(\mathrm{ir})^{2}-\Pi((\mathrm{i}-1) \mathrm{r})^{2}}{\Pi \mathrm{R}^{2}}=\frac{\mathrm{r}^{2}(2 \mathrm{i}-1)}{\mathrm{R}^{2}}
$$

As traffic from nodes in outer rings traverse to inner ring nodes towards gateway node. Traditional routing algorithms like minimum cost routing focus on choosing a route with least cost in terms of number of hops, distance, energy, etc. But, are not suitable for uniform traffic distribution and hence to prolong network lifetime. Here network lifetime is defined as time duration when network first node fails due to lack of energy.

When, network follows a routing algorithm which can distribute the traffic load uniformly across nodes (Li et al., 2013), then traffic at $\mathrm{i}^{\text {th }}$ ring nodes can be computed by (26).

$$
\Delta_{\mathrm{i}}=\frac{1}{\mathrm{~N}_{\mathrm{i}}} \Sigma \mathrm{j}=\mathrm{i} \ldots \mathrm{n}\left(\mathrm{N}_{\mathrm{j}} \cdot \beta . \mu_{\mathrm{j}}\right)
$$

As nodes are uniformly distributed then average number of peer links is given by (27).

$$
\mathrm{k}=\left[\frac{\mathrm{N} \cdot \Pi \cdot \mathrm{r}^{2}}{\Pi \cdot \mathrm{R}^{2}}\right]
$$

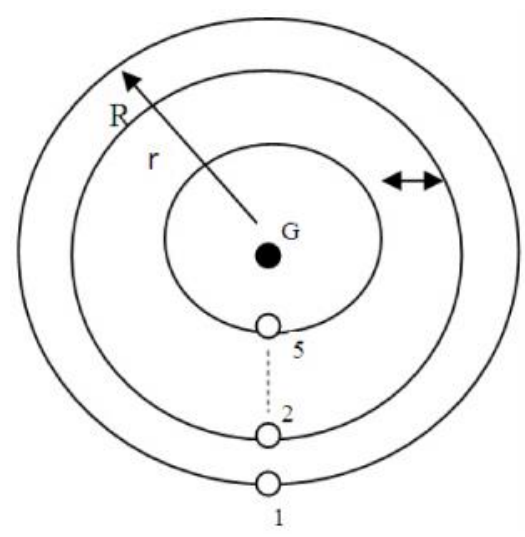

Fig. 12: Circular network area divided into rings

Under uniform load distribution, by law of flow conservation (28) average load at link (j,i) and (i,l) can be assumed as $\mathrm{t}_{\mathrm{i}, \mathrm{j}}=\frac{2 \Delta_{\mathrm{i}}}{\mathrm{k}} \forall \mathrm{j}=1 \ldots \mathrm{k} / 2$. Assuming traffic is dispersed in forward direction only. A node can go to sleep after receiving his share of traffic.

$$
\sum_{\mathrm{j}=1}^{\mathrm{k} / 2} \mathrm{f}_{\mathrm{j}, \mathrm{i}}=\sum_{\mathrm{l}=1}^{\mathrm{k} / 2} \mathrm{f}_{\mathrm{i}, 1}=\Delta_{\mathrm{i}}
$$

Data transmission between two nodes ' 1 ' and ' 2 ' (Fig. 12) at $\mu_{\mathrm{i}}=30$ is illustrated in Fig. 13 using parameters in Table 2 and table 3. A batch of 30 packets requires $140.331 / 4 \mathrm{~s}$. Remainder $X=9.86 \mathrm{~ms}$ at node ' 1 ' and Reminder $Y=4.86 \mathrm{~ms}$ at node ' 2 ' are less than respective awake window, so node will stay in awake state till end of awake window.

\section{Numerical Results and Discussion}

Actual values of parameters in (24) vary from one hardware implementations of radio to other.

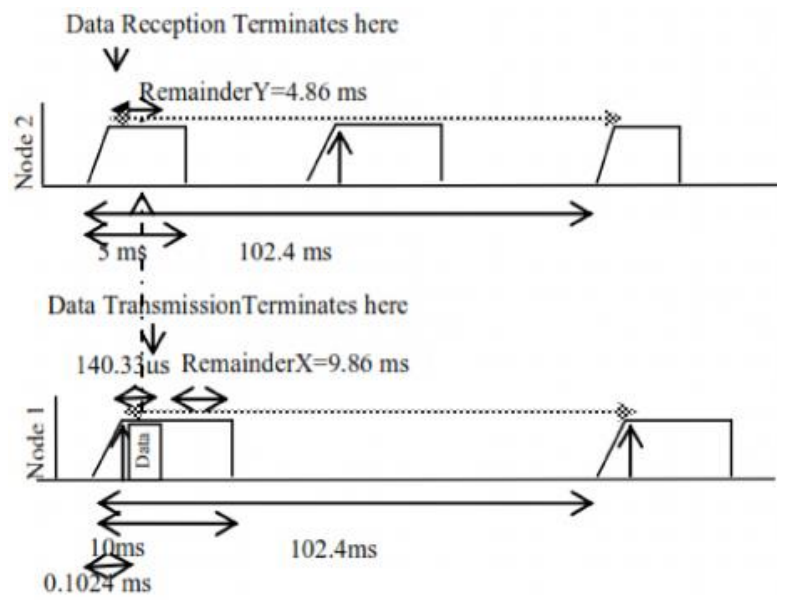

Fig. 13: Data communication between two nodes 
Table 2: Parameters value used in present energy model

\begin{tabular}{|c|c|c|}
\hline Symbol & Description & Value \\
\hline $\mathrm{F}$ & Frequency synthesizers settling time & 150 \\
\hline I & Frequency synthesizers current drawn & $24 \mathrm{~mA}$ \\
\hline $\mathrm{V}$ & $\begin{array}{l}\text { Frequency synthesizers voltage drawn } \\
2.5 \mathrm{~V} \mathrm{E}_{\mathrm{LA}} \text { Switch energy }\end{array}$ & $1.81 / 4 \mathrm{~J}$ \\
\hline A & Beacon Interval & $102.4 \mathrm{~ms}$ \\
\hline $\mathrm{R}_{\mathrm{awk}}$ & $\begin{array}{l}\text { Energy consumption in listening beacon } \\
\text { M Safety Margin } \\
E_{D} \text { Circuit energy consumption } 0.740 \text { watt }\end{array}$ & $\begin{array}{l}0.0037 \mathrm{~J} \\
0.1024 \mathrm{~ms}\end{array}$ \\
\hline $\mathrm{S}$ & Beacon size & $\begin{array}{l}162 \text { to } \\
2246 \\
\text { bytes }\end{array}$ \\
\hline$P_{t}$ & Receiving Power & 0.9 watt \\
\hline $\mathrm{P}_{\mathrm{r}}$ & Transmission Power & 1.33 watt \\
\hline W & Awake window size & $10 \mathrm{~ms}$ \\
\hline $\mathrm{L}$ & Listen Interval to peer beacon & $5 \mathrm{~ms}$ \\
\hline $\mathrm{D}_{\mathrm{awk}}$ & Energy consumption in Awake window & $0.0074 \mathrm{~J}$ \\
\hline $\mathrm{C}_{\text {slot }}$ & Contention Slot & $10 \mu \mathrm{s}$ \\
\hline $\mathrm{C}_{\min }$ & Contention window size & 15 \\
\hline $\mathrm{P}_{\mathrm{S}}$ & Size of packet & 256 bytes \\
\hline DIFS & DCF Inter-frame Space & $41 \mu \mathrm{s}$ \\
\hline B & Bandwidth & $\begin{array}{l}11,54,128 \\
\text { Mbps }\end{array}$ \\
\hline $\mathrm{O}$ & Observation period & 1 second \\
\hline DTIM & Delivery traffic indication map period & 2 \\
\hline
\end{tabular}

Table 3: Parameters value used in circular network area

\begin{tabular}{lll}
\hline Symbol & Description & Value \\
\hline $\mathrm{N}$ & $\begin{array}{l}\text { Number of nodes in circular area 50,100 } \\
\mu_{\mathrm{i}} \text { Poisson trafficrateper beacon }\end{array}$ & $\begin{array}{l}30,50, \\
100\end{array}$ \\
& & $\frac{1}{5}$ \\
$\beta$ & Density of MAP & 500 meter \\
$\mathrm{R}$ & Radius of circular area & 100 meter \\
$\mathrm{r}$ & Radius of circular ring & 500 joules \\
$\mathrm{e}$ & Initial energy of nodes
\end{tabular}

Considering frequency synthesizers by (Terrovitis et al., 2004), which draws $11 \mathrm{~mA}$ and $24 \mathrm{~mA}$ from the $3.3 \mathrm{~V}$ and $2.5 \mathrm{~V}$ supplies correspondingly and having settling time <150us. Various parameters used in calculations are listed in Tables 2 and 3. The proposed model of IEEE $802.11 \mathrm{~s}$ has been analysed in
MATLAB. Results for network having nodes in light and deep sleep mode with beacon size of 162 bytes have been computed (Figs. 14-26).

For deep insight due to impact of number of peer links, a scenario of no traffic has been considered initially. Analysis reveals that as number of peer links increases, node devotes more time in idle state and energy consumption increases (Fig. 14.). In light sleep mode, with bandwidth 54Mbps, DTIM=2, and network nodes having one, two, three, four, five, ten, and fifteen number of peer links have been evaluated. It is found that in spite of no traffic, energy saving is $90.16 \%$, $85.25 \%, 80.33 \%, 75.42 \%, 70.52 \%, 45.95 \%, 21.4 \%$ respectively. With nineteen peer links, a node remains in fully awake state. In deep sleep mode, in spite of number of peer links, energy saving remains constant at $95.16 \%$. So, denser WMN network are less energy

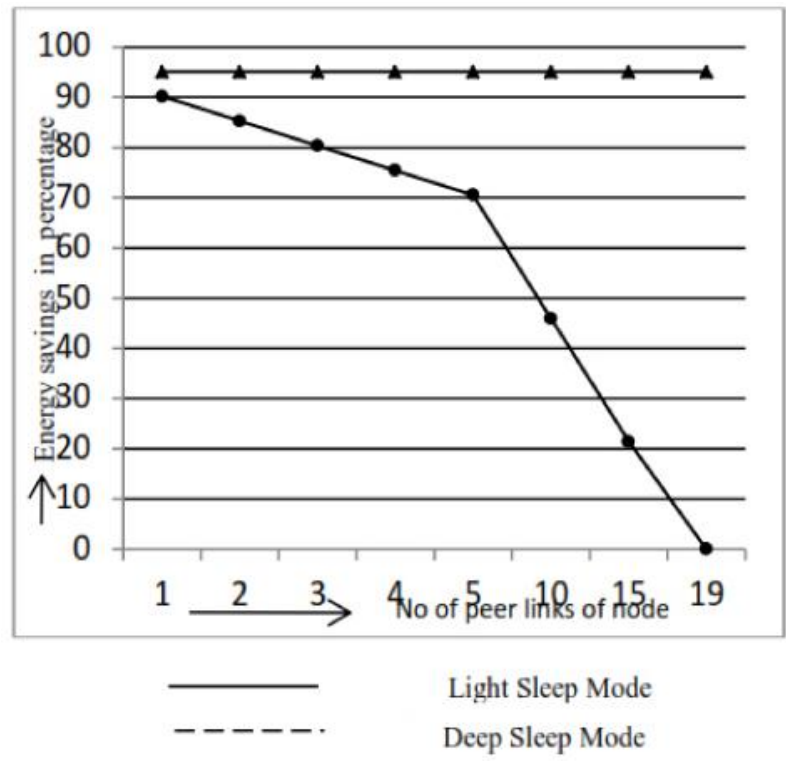

Fig. 14: Energy savings vs. peer links in power save mode in void traffic

efficient in light sleep mode. Further, it can be easily figured-out that with beacon size energy saving reduces. For each addition of peer node energy saving reduces by $\sim 4.91 \%$ in light sleep mode.

Moreover, higher bandwidth is more energy efficient due to decrease in energy consumption in receiving and transmitting of beacons or traffic (Fig. 15). Results have been observed for DTIM=2, and four peer link. Energy savings for bandwidth $11 \mathrm{Mbps}$, $54 \mathrm{Mbps}, 128 \mathrm{Mbps}$ are 74.81\%, 75.42\%, 76.47\% respectively. Every increase of $1 \mathrm{Mbps}$ in bandwidth 


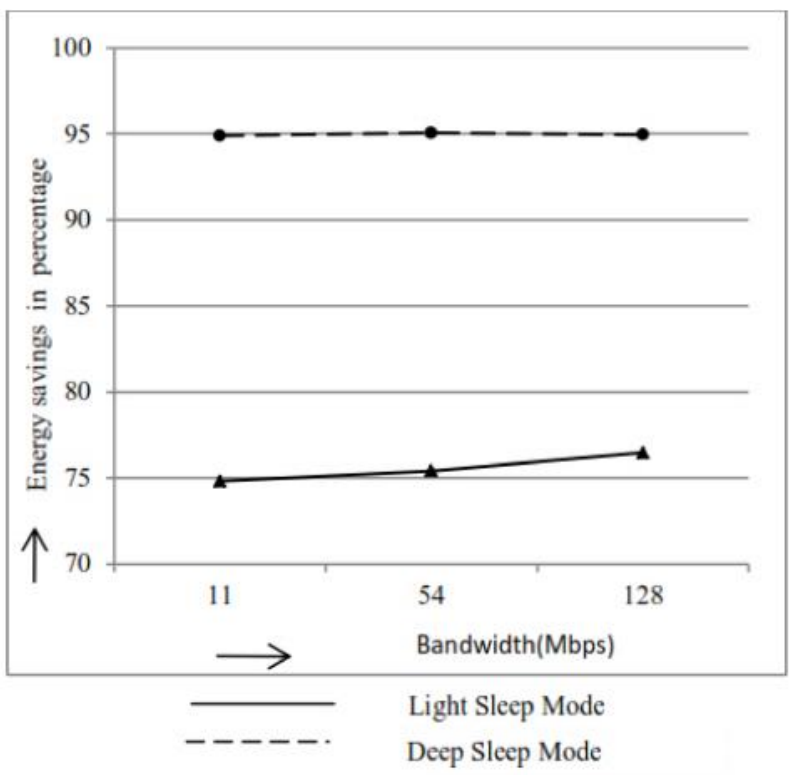

Fig. 15: Energy savings vs. bandwidth in power mode in void traffic

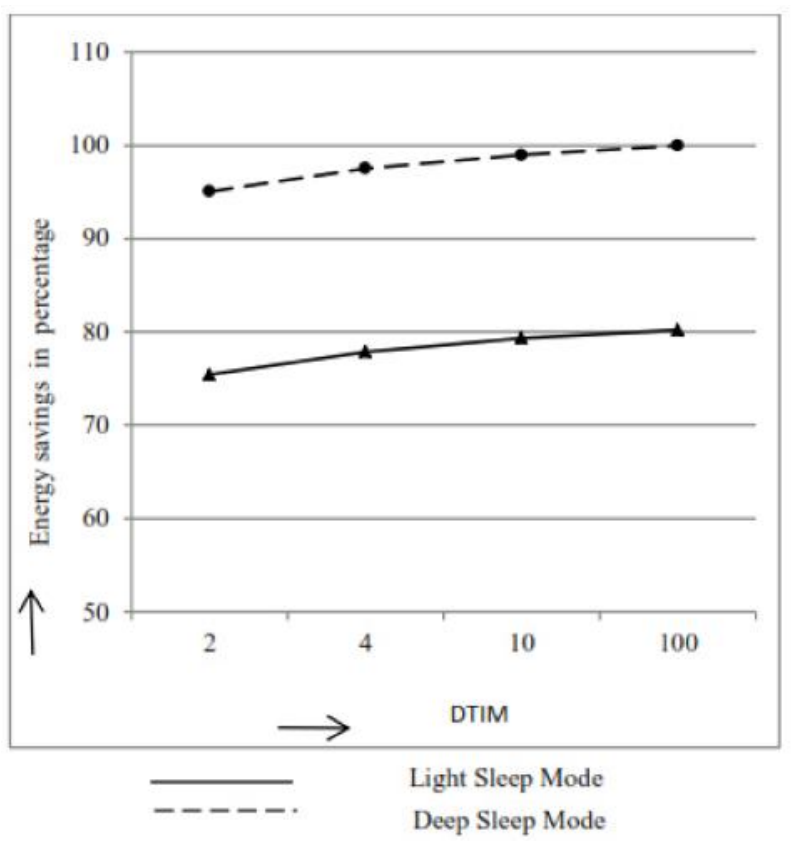

Fig. 16: Energy savings vs. DTIM in light sleep mode and deep sleep mode in void traffic

enhances energy saving by $\sim 0.0142 \%$ in light sleep mode.

Further, incrementing DTIM period also enhances energy savings. At bandwidth $54 \mathrm{Mbps}$ and four number of peer links, in light sleep mode energy savings for $\mathrm{DTIM}=2$, DTIM $=4, \mathrm{DTIM}=10$, DTIM=100 are $75.42 \%, 77.86 \%, 79.33 \%, 80.2 \%$

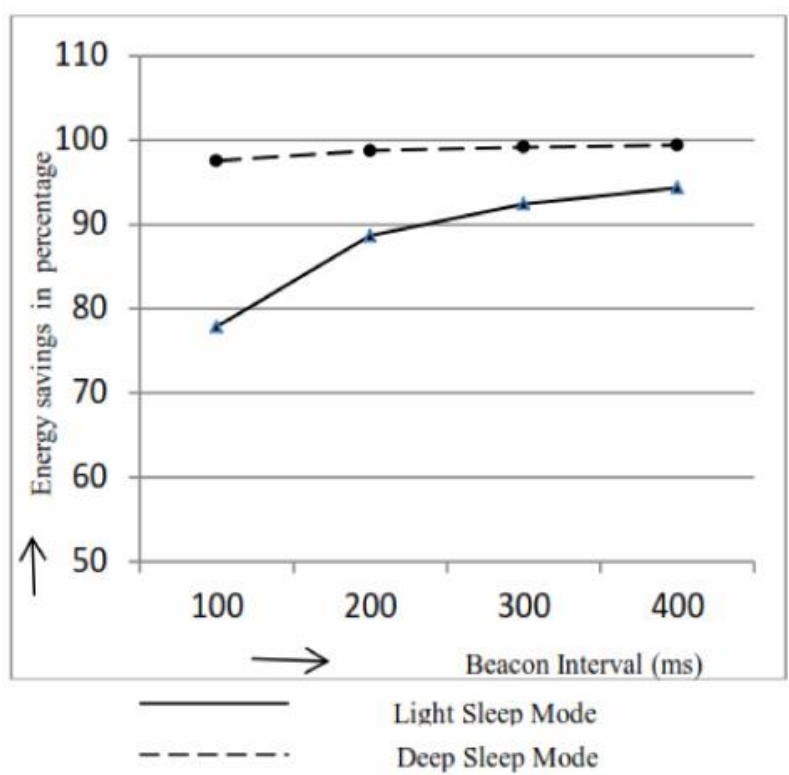

Fig. 17: Energy savings vs. Beacon Interval in light and deep sleep mode in void traffic

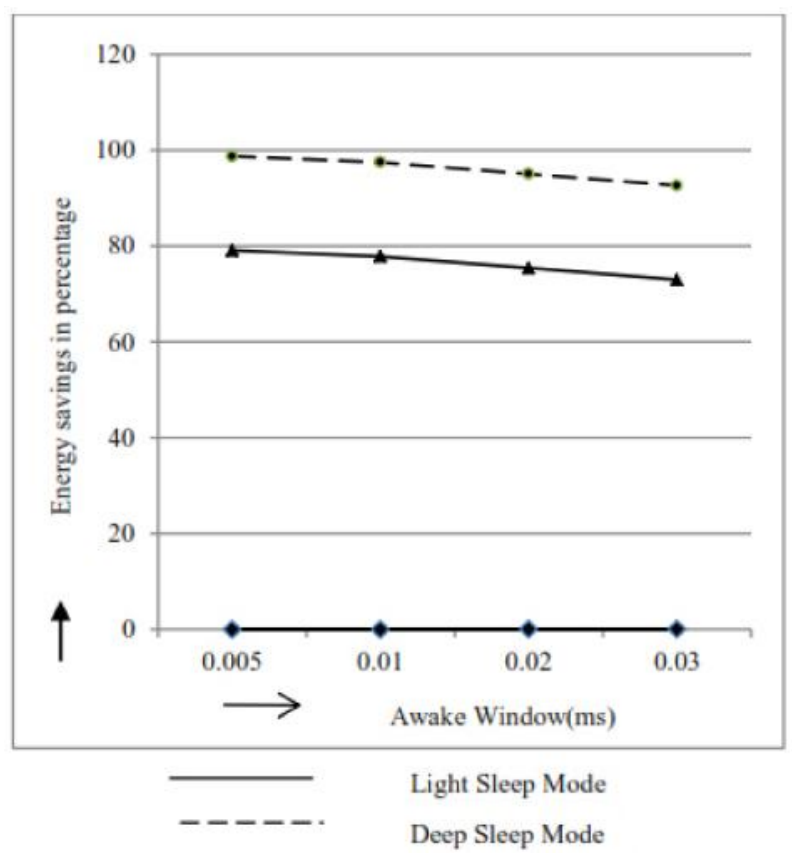

Fig.18: Energy savings vs. Awake Window in active mode, light sleep mode and deep sleep mode in void traffic

respectively (Fig. 16). While in deep sleep mode it gradually enhances as $95.07 \%, 97.52 \%, 98.98 \%$, and $99.99 \%$. The Beacon containing DTIM indicates presence of multicast/broadcast traffic. The higher value of DTIM period, suggests for deep sleep mode of links during low traffic. So by setting deep sleep mode for redundant peer links, additional energy can be saved. Energy saving versus Beacon Interval and 


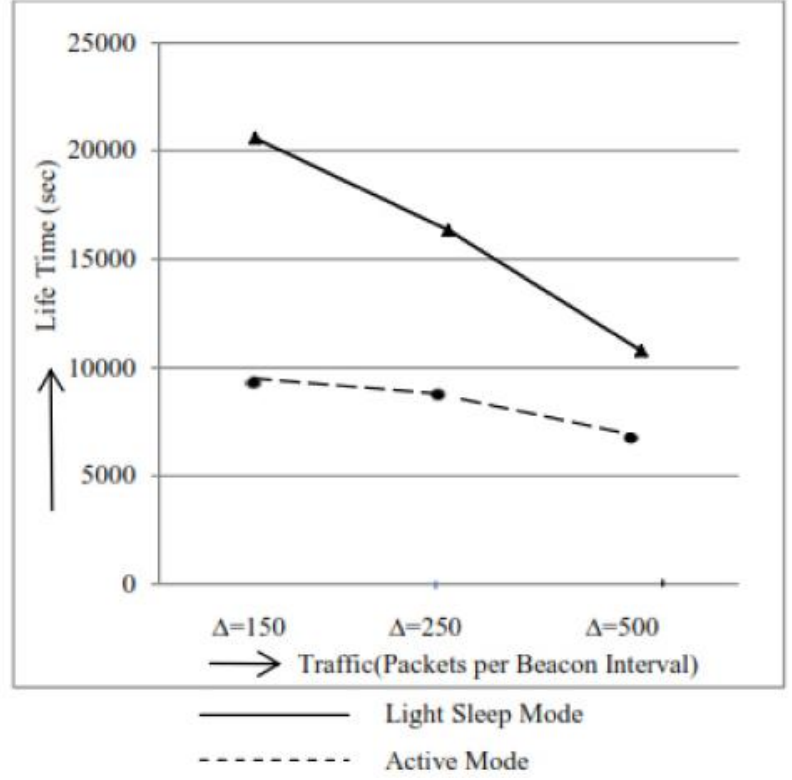

Fig. 19: Node life time in first ring, N=50, DTIM =2, bandwidth=54 Mbps

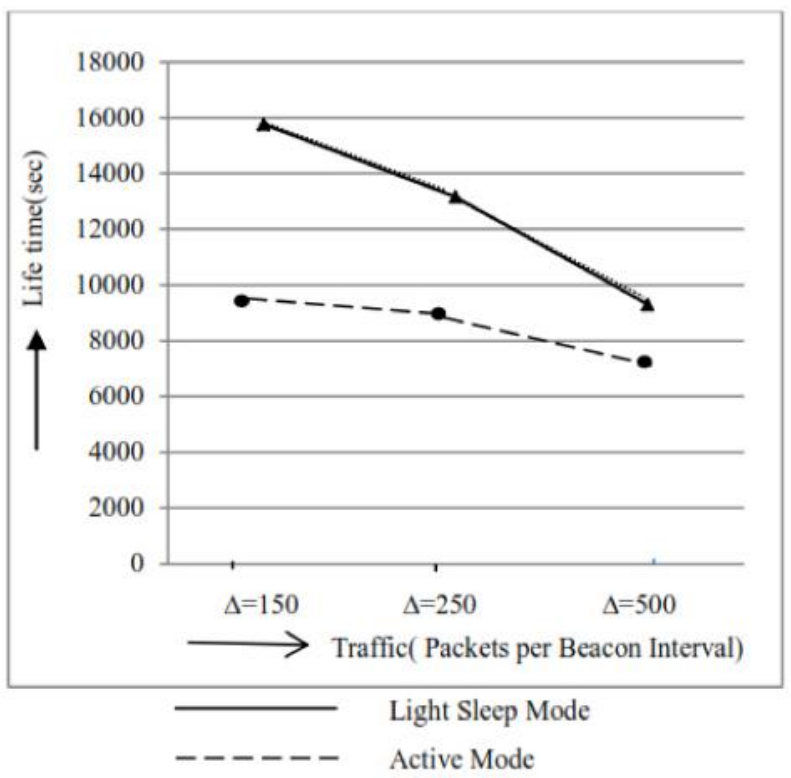

Fig. 20: Nodes life time in first ring, $\mathrm{N}=100$, DTIM $=2$, bandwidth $=54$ Mbps

four peer link is given in Fig. 17. Increasing Beacon Interval save energy consumption but increases the delay as shown in Fig. 25. Impact of Awake Window size is also shown in Fig. 18. For every increase of 10 $\mathrm{ms}$ in Awake Window size, a $2.44 \%$ increases in energy consumption has been observed.

In contention based WMN and under traffic load,

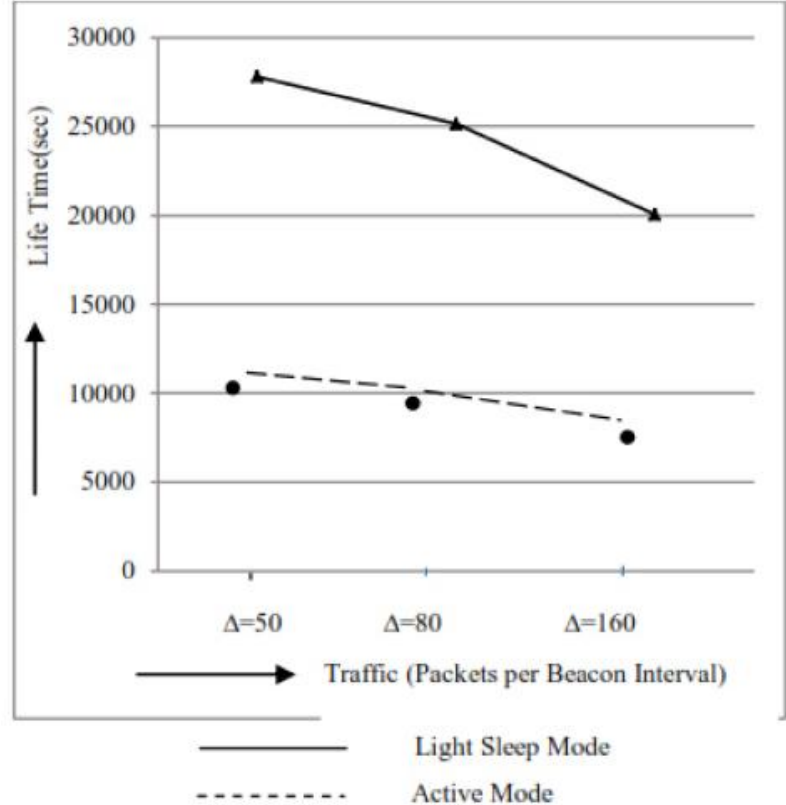

Fig. 21: Nodes life time in second ring, $N=100$, DTIM =2, bandwidth=54 Mbps

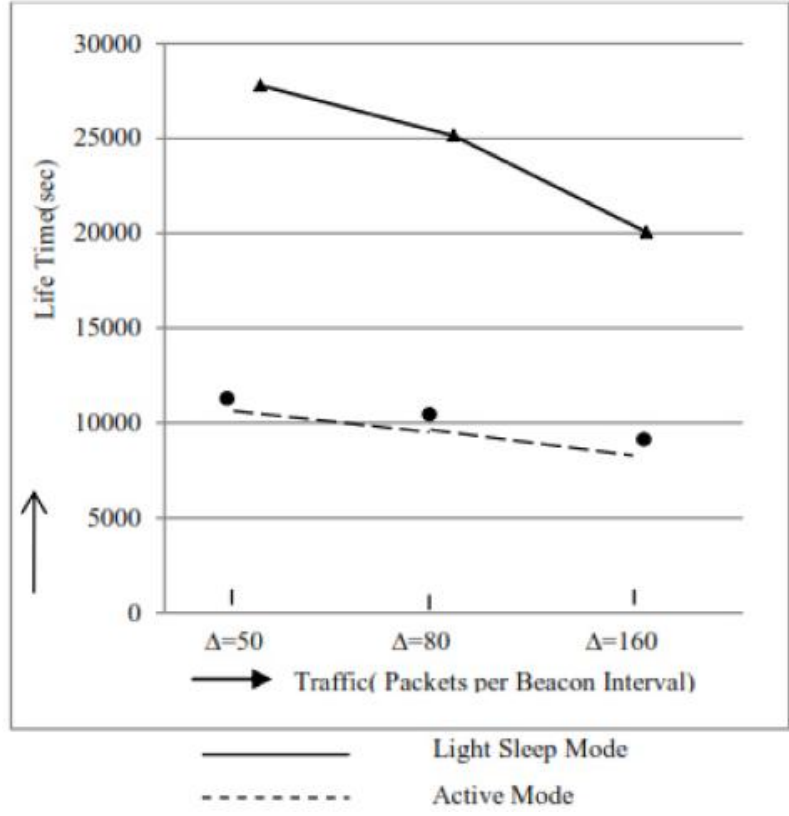

Fig. 22: Nodes life time in second ring, $\mathrm{N}=50$, DTIM $=2$, bandwidth $=54$ Mbps

network life time analyses for uniform distributed node in circular area are given in Figs. 19 to 22. Work can be easily extended for other topology and traffic distribution as well. Similarly for TDMA based WMN results can be computed easily. Two cases for 100 and 50 nodes have been considered. Traffic load increases as we move towards inner rings i.e., nodes in first ring are more loaded than in second ring. 
Lifetime of outer ring nodes (Figs. 21 and 22) is higher than inner one (Figs. 19 and 20). Network life time is upper bounded by nodes in $1^{\text {st }}$ ring. Life time of nodes in first and second ring in active mode vs. PSM has been evaluated. With increase in traffic arrival rate light sleep node devotes more in communication mode, so overall network life time decreases. With $\leq 150$, an improvement of $\sim 50 \%$ in network lifetime has been observed. In first ring beyond " $=750$ light sleeping nodes needs to stay in active mode. Network with less density of nodes (Figs. 19 and 22) have more life time than counter case due to less exchange of control packets/data with peer nodes.

Further, in analysis safety margin and awakewindow duration are assumed as $0.1024 \mathrm{~ms}$ and 10 $\mathrm{ms}$ respectively. Thus, the maximum of sleep possible within maximum sleep duration within a beacon interval comes out $92.3(102.4 \mathrm{~ms}-10 \mathrm{~ms}-0.1024 \mathrm{~ms}=$ $92.3 \mathrm{~ms}$ ). For a steady state network with packet arriving at a regular rate and departure in order of arrival, this consequently determines delay of packets at a node due to PSM. As traffic load increases beyond 750 packets, delay increases sharply due to accumulated packets in queue (Fig. 23). Reported result of delay vs. Traffic by Bauschert T. and Porsch M. (2012) is given in Fig. 24 for comparison.Delay analysis with Beacon Interval is given in Fig. 25. Active mode does not have impact due to Beacon Interval.

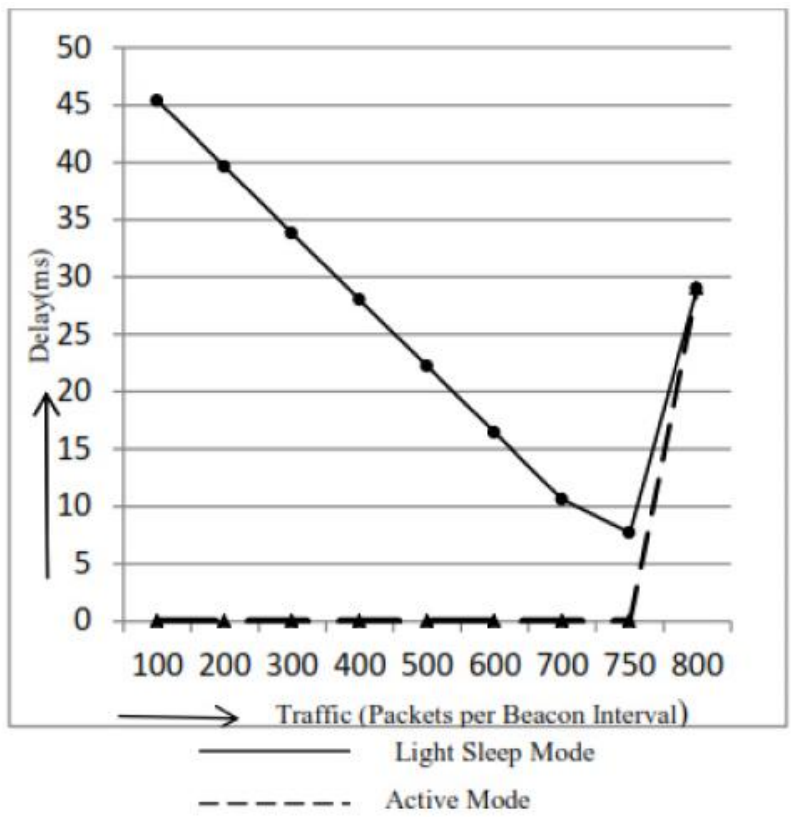

Fig. 23: Average delay vs. Traffic

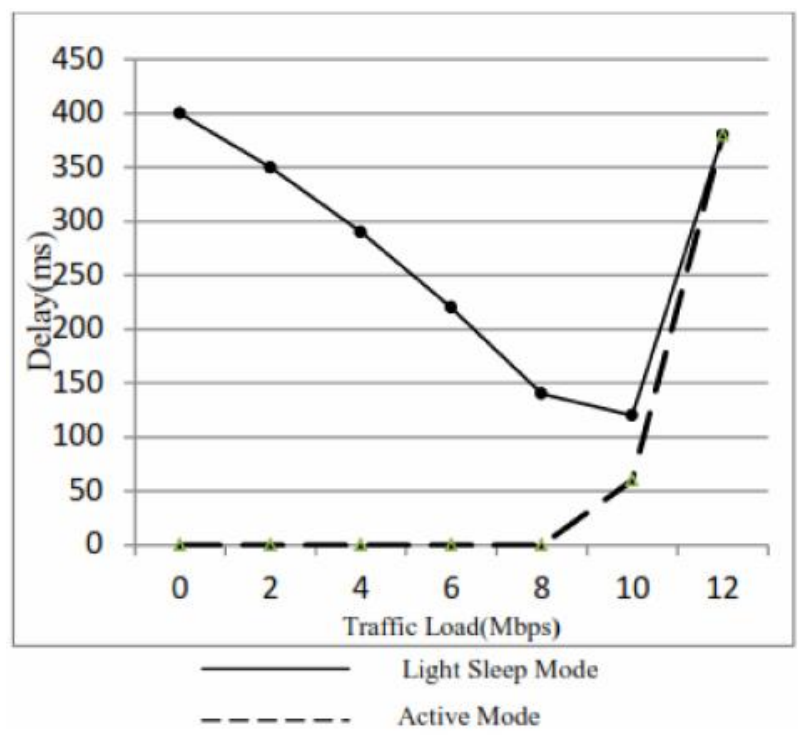

Fig. 24: Delay vs. traffic at 800 Beacon Interval

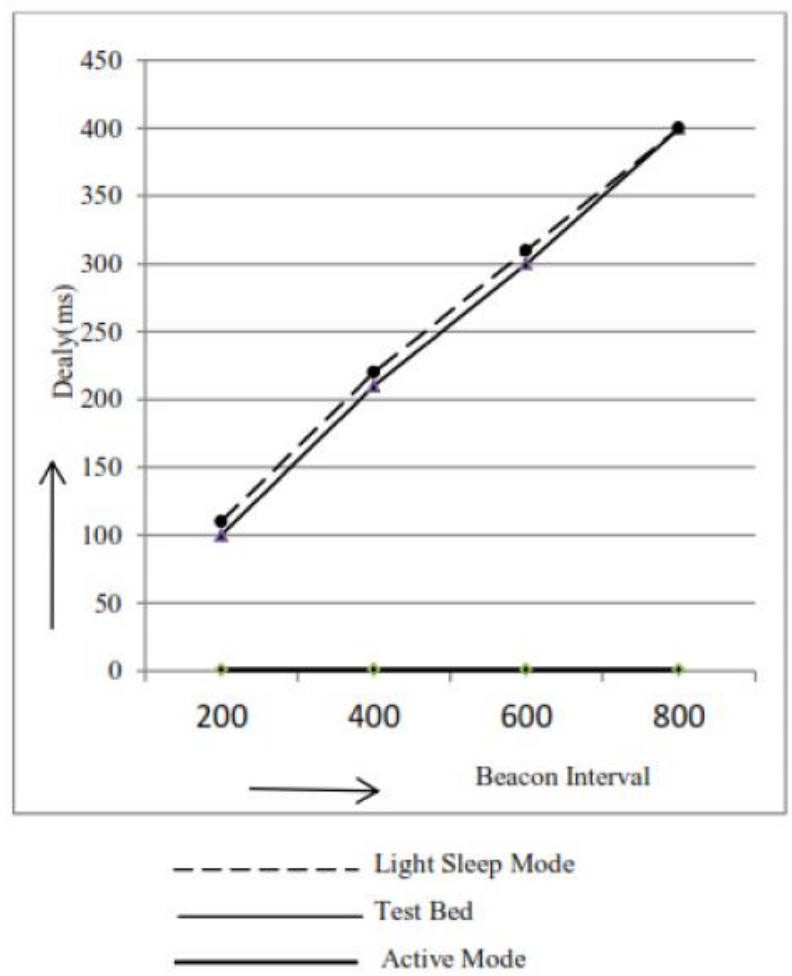

Fig. 25. Average delay vs. Beacon Interval

Further, if Queue buffer capacity is sufficient enough to accommodate traffic during sleep mode, typically during Beacon Interval. The power save mode does not have much impact on throughput of network at higher Beacon Interval (Fig. 26). At lower Beacon Interval due to over head in beacon exchange, a slight degradation in throughput has been observed. With 

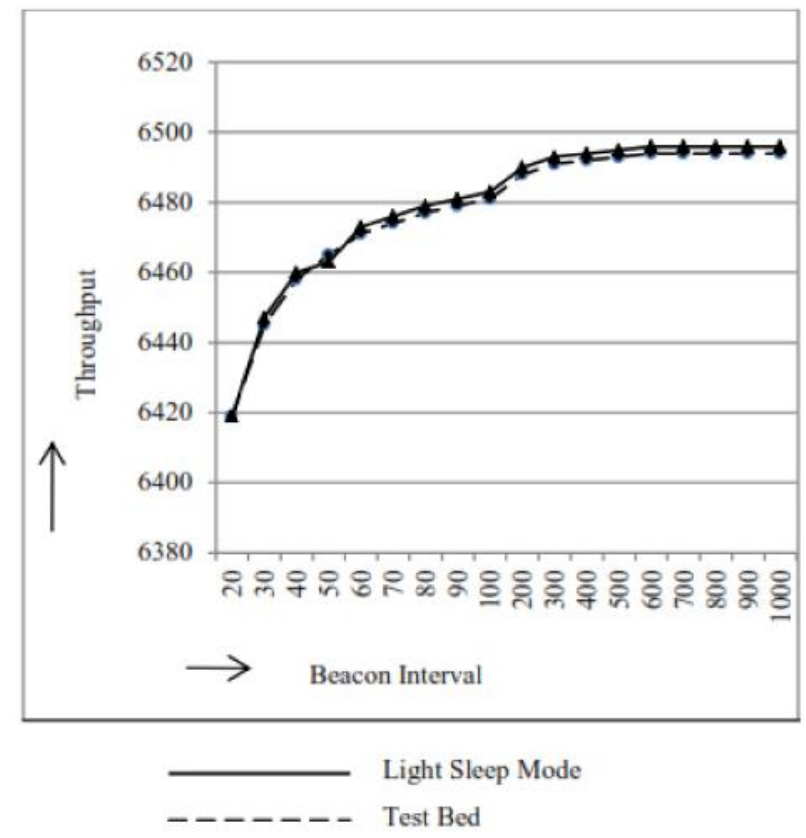

Fig. 26: Throughput vs. Beacon Interval

increase in traffic load a node stay in awake state for communication. During low traffic period, maximum redundant links can be set in deep sleep mode for further energy save.

\section{Comparison with Existing Work}

In (Bauschert and Porsch, 2013), for device driver Netgear WNDR3800 and TP-Link TL-MR3020, energy consumption results for increasing peer links has beenanalysed. Energy consumption increases linearly with addition of each peer. It is found that, in Netgear WNDR3800 and TP-Link TL-MR3020 energy consumption increases by $1.17 \%$ and $2.24 \%$ respectively at each addition. Network needs to be in fully awake state at 84 and 42 peers. It assumes beacon interval $1024 \mathrm{~ms}$, and awake window $102.4 \mathrm{~ms}$. Other parameters like beacon size, bandwidth, listen interval, etc. are not specified. In our proposed model, after re-evaluating results for beacon interval 1024 ms, awake window $102.4 \mathrm{~ms}$ as in (Bauschert and Porsch, 2013), shows linear increase of $0.49 \%$, and $0.51 \%$ energy increases for $11 \mathrm{Mbps}$, and $54 \mathrm{Mbps}$ respectively. Network remains fully awake at approximately 192 peers. This difference between our and reported test bed results is due to different hardware and measuring setup assumptions.

Furthure, Bauschert T. and Porsch M. (2012) have given test bed analysis for delay vs., traffic load, delay vs. Beacon Interval and throughput vs. Beacon Interval in power save mode of IEEE 802.11s. But energy measurement analysis has not been considered.The subsequent work of (Bauschert and Porsch, 2013) gives energy consumption analysis versus number of peer links only in test bed environment. Energy consumption for other parameters like traffic load, DTIM period, bandwidth, etc. has been not considered. The computed results for peer links scalability (Fig. 14), average delay vs., traffic load (Fig. 23), average delay vs. Beacon Interval (Fig. 25) and throughput (Fig. 26) have been found to follow the test bed observation of Bauschert T. and Porsch M. (2013) and (2012) respectively. The proposed model is in obedience with the standard, and can be useful in scientific field. Various available simulators like Qualnet, ns3, etc. have provision for various features of IEEE 802.11s except PSM. So it is hard to evaluate PSM of IEEE 802.11s by using different simulators. The proposed mathematical model prepares the way for better control of a system to choose optimal configuration parameters and to switch to PSM.

\section{Conclusion and Future Work}

This work represents analysis of power save mode of IEEE $802.11 \mathrm{~s}$ in aspects of energy efficiency, delay, and throughput. Numerical results prove that, in spite of no traffic with growth in peer links the energy consumption increases. For each addition of peer node energy consumption increases by $\sim 4.91 \%$ in light sleep mode. By opting deep sleep state for redundant peer links energy saving can be improved. Similarly, for each increase of $1 \mathrm{Mbps}$ in bandwidth, energy saving enhances by $\sim 0.0142 \%$ in light sleep mode. Providing higher bandwidth at each node may not be feasible. But using more bandwidth for links connecting to gateway can save more energy. Similarly, the increase in beacon size also enhances energy consumption. Moreover, higher Delivery Traffic Indication Map (DTIM) period also enhances energy savings. Additional energy can be saved by setting deep sleep mode for redundant peer links. For each increase of $10 \mathrm{~ms}$ in Awake Window size, a $2.44 \%$ increases in energy consumption has been observed. With increase in traffic arrival rate light sleep node devotes more in communication mode, so overall network life time decreases. By deploying nodes with more battery capacity or increasing density of nodes near gateway 
node can help prolong overall network lifetime. Queue buffer capacity should be sufficient enough to accommodate traffic during sleep mode and else may degrade throughput. With sufficient queue buffer at each node, except at lower Beacon Interval, the throughput of network does not degrade due to power save mode. In steady state system average delay is higher at low traffic rate. The traffic delay decrease gradually with higher traffic. But after a node reaches in fully awake stage, delay starts increasing again.

The analysis suggests tuning the network configuration to minimize energy consumption due to idle peer listening. The proposed model for analysis of PSM of IEEE 802.11s is in obedience with the standard, and can be useful in scientific field. The

\section{References}

IEEE Standard for Information Technology_-Telecommunications and information exchange between systems-Local and metropolitan area networks-(2011) Specific requirements Part 11: Wireless LAN Medium Access Control (MAC) and Physical Layer (PHY) specifications Amendment 10: Mesh Networking, IEEE Std 802.11s-2011, pp. 1-372

Swain P, Chakraborty S, Nandi S, and Bhaduri P (2015) Performance Modeling and Analysis of IEEE 802.11 IBSS PSM in Different Traffic Conditions IEEE Transactions on Mobile Computing 14 1644-1658

Aydogdu C and Karasan E (2011) An Analysis of IEEE 802.11 DCF and Its Application to Energy-Efficient Relaying in Multihop Wireless Networks IEEE Transactions on Mobile Computing 10 1361-1373

Jangsoo Lee and Sungchun Kim (2009) Mathematical system modeling and dynamic resource allocation through Kalman Filter based prediction in IEEE 802.11 PSM Industrial Technology IEEE International Conference on, Gippsland, VIC, pp. 1-6

Kindt P H, Saur M, Balszun M and Chakraborty S (2018) Neighbor Discovery Latency in BLE-Like Protocols IEEE Transactions on Mobile Computing 17 617-631

Miao G, Azari Aand HwangT(2016) E² -MAC: Energy Efficient Medium Access for Massive M2M Communications IEEE Transactions on Communications 64 4720-4735

Shahin N, Ali R and Kim Y T (2018) Hybrid Slotted-CSMA/CATDMAfor Efficient Massive Registration of IoT Devices proposed mathematical model prepares the way for better control of a system to choose optimal configuration parameters and to switch to PSM. Analysis suggests setting the best possible value for Ad hoc Traffic Indication Message (ATIM) window size, DTIM period, Beacon Interval, Queue Size, Bandwidth, and PSM towards peer links etc. to minimize energy consumption during idle peer listening. Optimal light and deep sleep mode of nodes can be selected for given traffic to support admissible throughput, and delay. Traffic shaping approaches to extend sleep duration of nodes can help in this direction. Various other criteria of PSM of link can be based on network repair requirements, battery power level, link quality, traffic load, etc.

\section{IEEE Access 6 18366-18382}

Rodenas-Herraiza D, Garcia-Sanchezb AJ, Garcia-Sanchezb F, Garcia-Harob J (2015) On the improvement of wireless mesh sensor network performance under hidden terminal problems Future Generation Computer Systems 45 95113

Zhu Y H, Luan S, Leung V C M and Chi K (2015) Enhancing Timer-Based Power Management to Support DelayIntolerant Uplink Traffic in Infrastructure IEEE 802.11 WLANs IEEE Transactions on Vehicular Technology 64 386-399

Purandare R G, Kshirsagar S P and Koli S M (2016) Analysis of Various Parameters for Link Adaptation in Wireless Transmission Innovations in Computer Science and Engineering. Advances in Intelligent Systems and Computing,. Springer, Singapore 413 9-19

Rahimi Malekshan K, Zhuang W and Lostanlen Y (2016) Coordination-based Medium Access Control With Spacereservation for Wireless Ad Hoc Networks IEEE Transactions on Wireless Communications 15 1617-1628

Lei L, Zhou J, Chen X, Qi L and Cai S (2012) Modelling and analysing medium access delay for differentiated services in IEEE 802.11s wireless mesh networks IET Networks 1 91-99

Carrano R C, Magalhaes LC S, Saade D C M and Albuquerque C V N (2011) IEEE 802.11s Multihop MAC: A Tutorial IEEE Communications Surveys \& Tutorials 13 52-67.

Sgora A, Vergados D D and Chatzimisios P (2009) IEEE 802.11s 
Wireless Mesh Networks: Challenges and Perspectives Mobile Lightweight Wireless Systems.Lecture Notes of the Institute for Computer Sciences, Social Informatics and Telecommunications Engineering 13 263-271

Bauschert T and Porsch M (2012) A Testbed Analysis of the Effects of IEEE 802.11s Power Save on Mesh Link Performance Information and Communication Technologies. Lecture Notes in Computer Science, Springer Berlin Heidelberg, pp. 1-11

Bauschert T and Porsch M (2013) A Testbed Evaluation of the Scalability of IEEE 802.11s Light Sleep Mode. Advances in Communication Networking Lecture Notes in Computer Science, Springer Berlin Heidelberg 292-297

Bauschert T and Porsch M (2014) Aligned Beacon Transmissions to Increase IEEE 802.11s Light Sleep Mode Scalability Advances in Communication Networking. Lecture Notes in Computer Science, Springer International Publishing, pp 173-184
Capone Antonio, Malandra Filippo and Sansò Brunilde (2012) Energy savings in Wireless Mesh Networks in a timevariable context Mobile Networks and Applications 17298 311

Ma J, Lou W and Li X Y (2014) Contiguous Link Scheduling for Data Aggregation in Wireless Sensor Networks IEEE Transactions on Parallel and Distributed Systems 25 16911701

Cui S, Goldsmith A and Bahai A (2005) Energy-constrained Modulation Optimization Journal of IEEE Transactions on Wireless Communications 4 2349-2360

Li F, He X, Chen S, Jiang L and Wang Y (2013) Traffic distribution of circular sailing routing in dense multihop wireless networks Journal of Tsinghua Science and Technology 18 220-229

Terrovitis M, Mack M, Singh K and Zargari M (2004) A 3.2 to 4 $\mathrm{GHz}, 0.251 / 4 \mathrm{~m}$ CMOS frequency synthesizer for IEEE 802.11 a/b/g WLAN Solid-State Circuits Conference, 2004 Digest of Technical Papers ISSCC.2004 IEEE International, 98-515. 